\title{
Is Isolated Aphasia Associated with Atrial Fibrillation? A Prospective Study
}

\author{
Carlijn P.A. Giesbers Peter J. Koehler Tobien H. Schreuder \\ Atrium Medical Centre, Heerlen, The Netherlands
}

Key Words

Atrial fibrillation $\cdot$ Isolated aphasia $\cdot$ Stroke

\begin{abstract}
Background: A cardioembolic source, usually atrial fibrillation (AF), is detected in $14-30 \%$ of strokes. If AF is found, anticoagulation therapy provides a substantial decrease of the risk of recurrent cerebrovascular ischemic events. AF is often paroxysmal, and extensive diagnostic procedures may be necessary to detect it in patients. Considering cost-effectiveness and patient burden, however, not every suspected patient can be thoroughly screened. Therefore, the identification of risk factors for AF may be helpful. Previous studies have identified isolated aphasia as a risk factor for AF as the cause of the stroke. These studies, however, were performed with small population samples, in a retrospective setting or focused on a specific subtype of aphasia. The aim of this observational study is to prospectively evaluate whether there is a relation between isolated aphasia and AF as the cause of cerebrovascular ischemia. Methods: All patients admitted to the Atrium Medical Centre, Heerlen, the Netherlands, with cerebrovascular ischemia or transient ischemic attack in the period of August 2009 to March 2010 or October 2013 to January 2014 were included. The patients were evaluated by a neurologist and admitted to the Brain Care Unit for 24-48 h. Medical history, physical examination and diagnostic results were entered in a database. A diagnosis of isolated aphasia was assigned at admission using the National Institutes of Health Stroke Scale (NIHSS). Presence of AF was determined using a 12-lead electrocardiogram (ECG) on admission and continuous ECG monitoring for 1-2 days. During admission, aphasia tests were done, notably the ScreeLing Test and the Boston Naming Test. Data were analyzed using Pearson's $\chi^{2}$ test, Fisher's exact test, the Mann-Whitney $U$ test and univariate and multivariate logistic regression analyses. Results: A total of 347 patients were included, of which 115 (33.1\%) met the criteria for aphasia, with 26 (7.5\%) meeting the criteria for isolated aphasia. Of all patients, 90 had a his-
\end{abstract}


tory of $\mathrm{AF}$ (66.7\%) or $\mathrm{AF}$ was found during hospitalization (33.3\%). Patients with $\mathrm{AF}$, in contrast to those without, tended to present more often with any type of aphasia ( $41.1 \mathrm{vs.} 30.4 \%$ ) and isolated aphasia (10.0 vs. $6.6 \%)$, but these differences were not significant. A significant relation was found between global aphasia and AF $(p=0.011)$. Patients with AF scored significantly lower on the ScreeLing Test alone and on both aphasia tests together $(p=0.034)$. A history of transient ischemic attack significantly increased the risk for isolated aphasia (OR 2.66, 95\% CI 1.09-6.47). Conclusions: A statistically significant relation between isolated aphasia and AF could not be established in this study. However, our results showed a tendency towards significance. Further studies, in which rhythm is observed for a longer period of time, may be required to confirm this hypothesis.

(C) 2014 S. Karger AG, Basel

\section{Introduction}

Cerebrovascular ischemia can have a high impact on a patient's quality of life. It has a great burden of disease and is rated second on a disease-related mortality list in Europe [1] and fourth in the United States [2]. Of all strokes, 14-30\% have a cardioembolic source [3], which is usually atrial fibrillation (AF) $[4,5]$. AF is associated with a higher risk of ischemic cerebrovascular events and more severe ischemic strokes [5-7]. Treatment with anticoagulants is superior to treatment with antiplatelets, with a reduced risk of approximately $30 \%$ [5]. In the absence of a cardioembolic source, anticoagulants are not indicated. Therefore, it is important to detect or rule out AF as the cause of the stroke.

As described in international protocols, patients presenting with an ischemic cerebrovascular event undergo a 12-lead electrocardiogram (ECG) on admission and ECG monitoring for approximately 24-48 h. Not all patients with (paroxysmal) AF are detected during this period [8]. Nevertheless, it is not desirable to extensively evaluate every patient with regard to the cost-benefit ratio and patient burden. Decisions should be made on an individual basis by evaluating risk factors.

Several studies have been undertaken to explore the relation between specific stroke symptoms and the presence of AF as the cause of the stroke. These studies demonstrated a positive relation between aphasia and AF [4, 9-13]. In 15-30\% of the patients, aphasia is present in the acute stage of stroke $[11,14]$. Isolated aphasia has a lower incidence, yet exact numbers are unknown. Some studies have suggested a positive relation between isolated aphasia and AF $[9,15,16]$. However, these studies were performed with a small population sample [16], in a retrospective setting [9] or focused on a specific subtype of aphasia [15, 16].

The aim of the present observational study is to evaluate whether there is a relation between isolated aphasia and $\mathrm{AF}$ as the cause of cerebrovascular ischemia in a prospective setting.

\section{Methods}

After approval by the local medical ethics committee, all patients admitted to the Atrium Medical Centre, Heerlen, the Netherlands, with cerebrovascular ischemia or a transient ischemic attack (TIA) in the period of August 2009 to March 2010 or October 2013 to January 2014 were included. Exclusion criteria were age $<18$ years and intracranial pathology other than ischemia. No double inclusions were made; if a patient presented with a recurrent ischemic event, only the data of the first admission were used. 
On admission, patients were evaluated by a neurologist. After history taking and examination, physical impairment was assessed with the National Institutes of Health Stroke Scale (NIHSS). A cranial computed tomography (CT) or magnetic resonance (MR) imaging was performed in all cases, and laboratory tests as well as an ECG were done. Thrombolysis was carried out if the patient met the criteria of our local stroke protocol (admission less than $4.5 \mathrm{~h}$ after stroke onset, NIHSS score $>2.5$, absence of hemorrhage on CT and absence of other contraindications). All patients were admitted to our Brain Care Unit for 24-48 h with continuous ECG monitoring and blood pressure measurements at regular intervals. Treatment with aspirin was initiated, which was switched to clopidogrel after 2 weeks unless a cardioembolic source was identified.

Additional tests were done if indicated. An unknown localization of ischemia was assessed with MR imaging. Duplex carotid ultrasonography was performed if ischemia was located in the anterior cerebral circulation. A cardiologist evaluated ECG abnormalities and if AF was detected, antiplatelets were switched to oral anticoagulants.

Presence or absence of aphasia was evaluated on admission using bedside language examination. Comprehension, repetition and naming were tested according to the NIHSS. In case of TIA with complete recovery of language, a diagnosis was made based on the patients' or, more often, their family's report. When possible, the ScreeLing Test and the Boston Naming Test were performed during hospitalization. Isolated aphasia was defined as any type of aphasia without other major deficits. Patients with only minor facial palsy or a drift of one extremity accompanying aphasia were included.

The medical histories of the patients were assessed. A history of TIA and/or ischemic cerebrovascular disease was noted as relevant neurological history. A history of angina pectoris, peripheral vascular disease, myocardial infarction, hypertension, AF and/or heart failure was referred to as relevant cardiovascular history. A relevant history of endocrine disease was assumed if hypercholesterolemia, type 2 diabetes and/or hypothyroidism were present.

The following variables were also recorded: left- or right-handedness, smoking, alcohol use, presence of familial cardiovascular disease, medication use (antiplatelet, anticoagulant, statin or antihypertensive drug), level of cholesterol, low-density lipoprotein, glucose and C-reactive protein, type of aphasia, presence of leukoariosis or recent ischemia on imaging, localization of ischemia, carotid stenosis/occlusion on ultrasound, MR or CT angiography, and finally whether carotid endarterectomy was performed.

At discharge, the Trial of Org 10172 in Acute Stroke Treatment (TOAST) classification was assigned. Furthermore, the patient's discharge destination (home or institution) was recorded. According to the TOAST classification, patients were divided into 4 groups. Group 1 included patients with ipsilateral internal carotid artery stenosis $>50 \%$ on ultrasound, CT or MR angiography. Group 2 included patients with cardioembolism as the most likely cause of the stroke. Patients were assigned to group 3 if they had a lacunar infarct and did not meet the criteria for group 1 or 2 . Group 4 included patients with a cortical infarct who did not meet the criteria for group 1 or 2 or if they met the criteria for both groups.

Analyses were performed with IBM SPSS Statistics software 21, 2012. Pearson's $\chi^{2}$ test and Fisher's exact test were used for analyses of two categorical variables. The Mann-Whitney $\mathrm{U}$ test was used for the comparison of categorical variables with scale variables. Odds ratios (OR) and 95\% confidence intervals (CI) were calculated using univariate and multivariate logistic regression analyses. For multivariate analyses, a univariate $p=0.15$ was used. Significance was set at the $\mathrm{p}=0.05$ level. 
Table 1. Patient characteristics

\begin{tabular}{lcccr}
\hline Variables & All $(\mathrm{n}=347)$ & AF $(\mathrm{n}=90)^{1}$ & No AF $(\mathrm{n}=257)$ & \multicolumn{1}{c}{$\mathrm{p}$} \\
\hline $\begin{array}{l}\text { Patient characteristics } \\
\text { Mean age, years }\end{array}$ & & & & \\
Male sex & $73.5(20.0-98.9)$ & $79.6(48.4-98.9)$ & $71.4(20.0-95.3)$ & $<0.001$ \\
Handedness & $150(43.2)$ & $33(36.7)$ & $117(45.5)$ & 0.114 \\
$\quad$ & & & \\
$\quad$ Right dominance & $216(62.2)$ & $45(50.0)$ & $171(66.5)$ & 0.410 \\
$\quad$ Unte dominance & $21(6.1)$ & $6(6.7)$ & $15(5.8)$ & 0.410 \\
Relevant neurological history & $110(31.7)$ & $39(43.3)$ & $71(27.6)$ & 0.006 \\
Relevant cardiovascular history & $98(28.2)$ & $31(34.4)$ & $67(26.1)$ & 0.129 \\
Relevant history of endocrine disease & $255(73.5)$ & $82(91.1)$ & $173(67.3)$ & $<0.001$ \\
Smoking & $132(38.0)$ & $36(40.0)$ & $95(37.4)$ & 0.656 \\
$\quad$ & & & \\
$\quad$ Yes & $92(26.5)$ & $16(17.8)$ & $76(29.6)$ & 0.065 \\
$\quad$ No & $236(68.0)$ & $64(71.1)$ & $172(66.9)$ & 0.065 \\
Alcohnol use $>1$ drink/day & $19(5.5)$ & $10(11.1)$ & $9(3.5)$ & 0.006 \\
Positive family history for CVD & $33(9.5)$ & $6(6.7)$ & $27(10.5)$ & 0.383 \\
\hline Medication & $100(28.8)$ & $21(23.3)$ & $79(30.7)$ & 0.766 \\
\hline Platelet aggregation inhibitor & $158(45.5)$ & $42(46.7)$ & $116(45.1)$ & 0.802 \\
Oral anticoagulant & $43(12.4)$ & $31(34.4)$ & $12(4.7)$ & $<0.001$ \\
Statin & $127(36.6)$ & $37(41.1)$ & $90(35.0)$ & 0.302 \\
Antihypertensive drug & $236(68.0)$ & $75(83.3)$ & $160(62.6)$ & $<0.001$ \\
\hline Laboratory testing & & & & \\
Cholesterol level, mmol/l & $4.5(2.0-9.0)$ & $4.4(2.0-7.8)$ & $4.6(2.2-9.0)$ & 0.328 \\
LDL level, mmol/l & $2.7(0.6-6.9)$ & $2.6(0.9-5.3)$ & $2.7(0.6-6.9)$ & 0.290 \\
\hline
\end{tabular}

Values represent $\mathrm{n}(\%)$ or mean (range). CVD = Cardiovascular disease; LDL = low-density lipoprotein.

${ }^{1}$ AF history or during admission.

\section{Results}

\section{Patient Characteristics}

A total of 347 patients were included, of which 115 (33.1\%) met the criteria for aphasia, with $26(7.5 \%)$ meeting the criteria for isolated aphasia. Of all patients, 90 had a medical history of AF (66.7\%) or AF was found during hospitalization (33.3\%). Table 1 shows the baseline characteristics for patients with and without AF. Patients with AF used oral anticoagulants (34.4 vs. $4.7 \%, \mathrm{p}<0.001$ ) and antihypertensive drugs ( 83.3 vs. $62.6 \%, \mathrm{p}<0.001$ ) significantly more often than those without AF. $91.1 \%$ of the patients with AF had a relevant cardiovascular history in comparison to $67.3 \%$ of those without AF. Patients with AF were significantly older $(\mathrm{p}<0.001)$, but no significant difference was found in terms of gender. With reference to clinical presentation, patients with $\mathrm{AF}$, compared to those without, presented more often with a cortical infarct (75.6 vs. $51.8 \%$ ) and a NIHSS score $>9$ (38.9 vs. $17.9 \%)$. Those with AF were less often discharged to their home $(\mathrm{p}<0.001)$ and more often referred to an institution (table 2). No significant difference was found in the mortality rate during hospitalization $(\mathrm{p}=0.139)$. Nine patients died from pneumonia, 6 from cerebral herniation, 2 from intracranial bleeding (one after thrombolysis and the other from hemorrhagic transformation), 2 from myocardial infarction and 1 from pulmonary hemorrhage. 
Table 2. Clinical presentation

\begin{tabular}{|c|c|c|c|c|}
\hline Variables & All $(n=347)$ & $\mathrm{AF}(\mathrm{n}=90)^{1}$ & No AF $(n=257)$ & $p$ \\
\hline Thrombolysis & $57(16.4)$ & 17 (18.9) & $40(15.6)$ & 0.464 \\
\hline NIHSS score $>9$ & $81(23.3)$ & $35(38.9)$ & $46(17.9)$ & $<0.001$ \\
\hline CT/MR imaging (acute cerebral infarct) & $87(25.1)$ & $22(24.4)$ & $65(25.3)$ & 0.873 \\
\hline \multicolumn{5}{|l|}{ Infarct location } \\
\hline Cortical & $201(57.9)$ & $68(75.6)$ & $133(51.8)$ & $<0.001$ \\
\hline Lacunar & $144(41.5)$ & $21(23.3)$ & $123(47.9)$ & $<0.001$ \\
\hline Duplex (ipsilateral occlusion/stenosis) & $47(13.5)$ & $8(8.9)$ & $39(15.2)$ & 0.252 \\
\hline Deceased & $20(5.8)$ & $8(8.9)$ & $12(4.7)$ & 0.139 \\
\hline Discharged home & $189(54.5)$ & $33(36.7)$ & $156(60.7)$ & $<0.001$ \\
\hline \multicolumn{5}{|l|}{ Aphasia } \\
\hline Aphasia & $115(33.1)$ & $37(41.1)$ & $78(30.4)$ & 0.062 \\
\hline Isolated aphasia & $26(7.5)$ & $9(10.0)$ & $17(6.6)$ & 0.294 \\
\hline Global aphasia & $65(18.7)$ & $25(27.8)$ & $40(15.6)$ & 0.011 \\
\hline Wernicke's aphasia & $3(0.9)$ & $1(1.1)$ & $2(0.8)$ & 1.000 \\
\hline Broca's aphasia & $47(13.5)$ & $11(12.2)$ & $36(14.0)$ & 0.670 \\
\hline Total ScreeLing score & $64.0(19-72)$ & $61.5(38-71)$ & $64.7(19-72)$ & 0.042 \\
\hline Total Boston Naming Test score & $148.6(11-180)$ & $141.7(63-177)$ & $150.5(11-180)$ & 0.059 \\
\hline $\begin{array}{l}\text { Boston Naming Test + ScreeLing } \\
\text { score }\end{array}$ & $212.6(43-252)$ & $203.2(105-246)$ & $215.2(43-252)$ & 0.034 \\
\hline
\end{tabular}

Values represent $\mathrm{n}(\%)$ or mean (range). ${ }^{1} \mathrm{AF}$ history or during admission.

\section{Aphasia}

Patients with AF tended to present more often with any type of aphasia (41.1 vs. 30.4\%) and isolated aphasia (10.0 vs. 6.6\%); however, these differences were not significant. Nine patients with isolated aphasia had a history of AF or were found to have AF during admission. A significant relation was found between global aphasia and AF $(p=0.011)$. None of the patients were diagnosed with transcortical motor or transcortical sensory aphasia. Patients with AF scored significantly lower on the ScreeLing Test (61.5 vs. 64.7) and had a lower score for both aphasia tests together $(\mathrm{p}=0.034)$. Only 123 patients finished both tests, but no significant differences between the tested and non-tested group regarding AF and isolated aphasia were noted. For the non-tested group, we noted the following reasons for non-testing: $45.2 \%$ went home before being tested, $8.9 \%$ could not speak/were mute, $4.9 \%$ were cognitively impaired, $2.9 \%$ had vision problems, $2.6 \%$ did not speak Dutch and $0.6 \%$ refused to be tested.

Table 3 presents the OR calculated for isolated aphasia and several patient characteristics. A history of TIA significantly increased the risk of isolated aphasia, and a history of AF also tended to increase the risk of isolated aphasia (OR 2.30, 95\% CI 0.95-5.57), but this was not significant. After multivariate analyses, history of AF was adjusted for age, heart failure and history of TIA. When adjusted for history of heart failure, history of AF became significant (OR 3.13, CI 1.25-7.83).

In table $4, \mathrm{OR}$ were calculated for $\mathrm{AF}$ with several characteristics of aphasia. Global aphasia was significant in univariate analysis (OR 2.10, $\mathrm{p}=0.012$ ). When adjusted for the total ScreeLing Test score, significance was absent $(p=0.097)$. 


\section{Cerebrovascular" \\ Diseases}

Table 3. Relationship of isolated aphasia with several characteristics

\begin{tabular}{l|l}
\hline Cerebrovasc Dis Extra 2014;4:165-173 \\
\hline DOI: $10.1159 / 000365410$ & $\begin{array}{l}\text { @ 2014 S. Karger AG, Basel } \\
\text { www.karger.com/cee }\end{array}$ \\
\hline
\end{tabular}

Giesbers et al.: Is Isolated Aphasia Associated with Atrial Fibrillation? A Prospective Study

\begin{tabular}{|c|c|c|}
\hline Variables (number of patients) & $\begin{array}{l}\text { Isolated aphasia } \\
(\mathrm{n}=26)^{1}\end{array}$ & $\mathrm{p}$ \\
\hline \multicolumn{3}{|l|}{ Univariate } \\
\hline Age & $1.03(0.99-1.07)$ & 0.122 \\
\hline Male sex (14) & $1.59(0.71-3.54)$ & 0.259 \\
\hline History of AF (8) & $2.30(0.95-5.57)$ & 0.065 \\
\hline AF during admission (3) & $0.56(0.16-1.91)$ & 0.351 \\
\hline History of heart failure (1) & $0.22(0.03-1.68)$ & 0.145 \\
\hline History of TIA (8) & $2.66(1.09-6.47)$ & 0.031 \\
\hline History of CVA (3) & $0.59(0.17-2.04)$ & 0.405 \\
\hline Type 2 diabetes (4) & $0.68(0.23-2.03)$ & 0.486 \\
\hline Smoking (4) & $0.47(0.16-1.40)$ & 0.172 \\
\hline Positive family history for CVD (8) & $1.10(0.43-2.80)$ & 0.839 \\
\hline Total ScreeLing score (10) & $1.00(0.93-1.08)$ & 0.955 \\
\hline Total Boston Naming Test score (10) & $1.01(0.98-1.04)$ & 0.448 \\
\hline \multicolumn{3}{|l|}{ Boston Naming Test + ScreeLing } \\
\hline score (10) & $1.01(0.99-1.03)$ & 0.523 \\
\hline TOAST 1 (3) & $1.06(0.30-3.72)$ & 0.925 \\
\hline TOAST 2 (8) & $1.45(0.61-3.47)$ & 0.402 \\
\hline TOAST 4 (15) & $2.46(1.10-5.54)$ & 0.029 \\
\hline Discharged home (24) & $11.35(2.64-48.8)$ & 0.001 \\
\hline \multicolumn{3}{|l|}{ Multivariate } \\
\hline \multicolumn{3}{|l|}{ History of AF, adjusted for } \\
\hline Age & $1.96(0.79-4.92)$ & 0.149 \\
\hline History of TIA & $2.29(0.94-5.60)$ & 0.068 \\
\hline History of heart failure & $3.13(1.25-7.83)$ & 0.015 \\
\hline Age + history of TIA & $2.01(0.80-5.06)$ & 0.138 \\
\hline Age + history of heart failure & $2.64(1.03-6.77)$ & 0.043 \\
\hline TIA + history of heart failure & $3.11(1.23-7.86)$ & 0.016 \\
\hline
\end{tabular}

Table 4. Relationship of AF with several characteristics of aphasia

\begin{tabular}{lll}
\hline Variables (number of patients) & $\mathrm{AF}(\mathrm{n}=90)^{1}$ & $\mathrm{p}$ \\
\hline Univariate & $1.60(0.98-2.63)$ & 0.063 \\
Aphasia (37) & $1.57(0.67-3.70)$ & 0.297 \\
Isolated aphasia (9) & $2.10(1.18-3.70)$ & 0.012 \\
Global aphasia (25) & $1.43(0.13-16.00)$ & 0.770 \\
Wernicke's aphasia (1) & $0.86(0.42-1.76)$ & 0.670 \\
Broca's aphasia (11) & $0.97(0.92-1.01)$ & 0.110 \\
Total ScreeLing score & $0.99(0.98-1.01)$ & 0.216 \\
Total Boston Naming Test score & $0.99(0.98-1.00)$ & 0.167 \\
Total Boston Naming Test + & & \\
$\quad$ ScreeLing score & & 0.097 \\
\hline $\begin{array}{l}\text { Multivariate } \\
\text { Global aphasia, adjusted for }\end{array} \quad$ Total ScreeLing score & & \\
\hline \multicolumn{1}{l}{${ }^{1}$ Values represent OR (95\% CI). } & \\
\hline
\end{tabular}



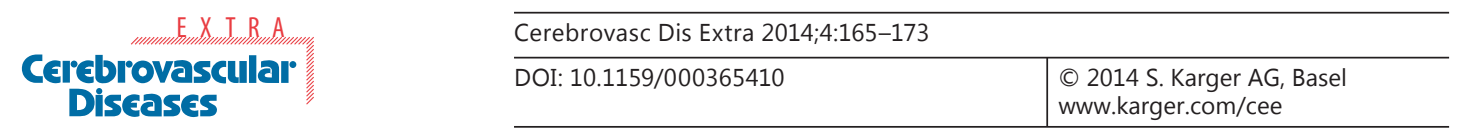

Giesbers et al.: Is Isolated Aphasia Associated with Atrial Fibrillation? A Prospective Study

\section{Discussion}

Our study did not show a significant relation between isolated aphasia and AF. We demonstrated a tendency towards significance, but our hypothesis could not be proven. A history of AF tended to have a stronger relation with isolated aphasia than AF detected during admission. A positive relation between global aphasia and AF and a tendency towards significance for aphasia in general and AF were observed. With regard to the aphasia tests performed, patients with AF scored significantly lower on the ScreeLing Test alone and on both tests together.

In agreement with previous studies, patients with $\mathrm{AF}$ were more severely impaired, less often discharged to their home and older than patients without AF $[9,12]$. The incidence of aphasia was similar to that reported in earlier studies, namely $33.1 \%$ in acute stage $[10,11$, 13]. The incidence of AF (25.9\%) was also comparable to other published data [3]. As in other studies, our results confirmed a significant relation between $\mathrm{AF}$ and the presence of a cortical infarct $[10,12]$.

In contrast to several other studies, our data did not show a significant relation between isolated aphasia and $\operatorname{AF}[9,15,16]$. There are two possible explanations for this discrepancy. First, our results are based on a relatively small number of patients. If more patients with isolated aphasia had been be included, a potential significant relation might have been detected. Second, the group of patients with isolated aphasia included significantly more patients classified as TOAST 4. Of those patients, 13 did not have AF on ECG monitoring or an ipsilateral internal carotid artery stenosis/occlusion on ultrasound. It is possible that (paroxysmal) AF was not detected in those patients and that a more extensive follow-up might have demonstrated more patients with AF [17]. The relation between isolated aphasia and AF might have been underestimated. Contrary to the retrospective study of Fennis et al. [9], no significant relation was found between isolated aphasia and gender, type 2 diabetes and smoking in our study. Our small population sample could be accountable for this.

In several studies, such as that by Arboix et al. [18], in-hospital mortality was found to be significantly higher in stroke patients with AF compared to those without AF. In our study, however, no significant difference was found in mortality during hospitalization between the two groups ( $p=0.139$ ). This may be due to our small sample size or because of the difference in length of hospitalization.

In our study, $34.4 \%$ of all patients with $\mathrm{AF}$ were treated with anticoagulants prior to admission. This is different from the number found by Lamassa et al. [12], who noted a use of anticoagulants in only $8.4 \%$ of patients. The fact that we included patients with a relevant neurological history could explain this difference. A cardioembolic origin of stroke could already have been established and treated. More advanced diagnostics and better adherence to international guidelines in recent times could also have accounted for part of the difference.

In a previous study by Capmany et al. [19], the underlying structural heart disease causing cardioembolism was determined by performing color Doppler echocardiography in over 400 cardioembolic stroke patients. AF was found in $79.1 \%$ of these patients, but isolated AF accounted for only $21.9 \%$ of strokes. A common structural finding associated with $\mathrm{AF}$ was hypertensive left ventricular hypertrophy. In our study, however, transesophageal echocardiography was not routinely used in all patients, since two-thirds of the AF patients had already been diagnosed with AF before having had an ischemic stroke. Therefore, we were unable to reliably distinguish between isolated $\mathrm{AF}$ or $\mathrm{AF}$ combined with another underlying potential source of cardioembolism [19].

Our study has some limitations. As mentioned above, our results are based on a relatively small sample size. Wide 95\% confidence intervals were present, and multivariate analyses could only be performed with three variables. Therefore, our results should be interpreted 
with caution. Another focus of attention is that only $34.9 \%$ of the patients completed both aphasia tests. Despite the fact that no significant differences were found regarding AF and isolated aphasia between the tested and non-tested group, results would be more reliable if a higher percentage of patients had been tested. Most of the missing patients were not tested because they went home or to a rehabilitation center before finishing the tests. In general, patients were admitted for a short period of time during which several additional diagnostics were performed. Hence, the lack of time was the most important reason patients went home before being fully tested.

In conclusion, this prospective observational study could not prove the hypothesis that isolated aphasia is related to cardioembolic stroke. Our results show a tendency towards significance but a larger sample size is needed. More prospective studies should be performed before a definite relation can be confirmed. Nonetheless, a cardioembolic source should be thoroughly evaluated when a patient presents with isolated aphasia without an evident cause.

\section{Disclosure Statement}

The authors have no conflicts of interest to disclose.

\section{References}

1 Nichols M, Townsend N, Scarborough P, Rayner M: European Cardiovascular Disease Statistics 2012: EuroHeart II, ed 4. European Heart Network and European Society of Cardiology.

2 Miniño AM, Murphy SL, Xu J, Kochanek KD: Deaths: final data for 2008. Natl Vital Stat Rep 2011;59:1-126.

-3 Arboix A, Alio J: Acute cardioembolic cerebral infarction: answers to clinical questions. Curr Cardiol Rev 2012; 8:54-67.

4 Kimura K, Minematsu K, Wada K, Yonemura K, Nakajima M: Clinical characteristics in transient ischemic attack patients with atrial fibrillation. Intern Med 2003;42:255-258.

5 Aguilar MI, Hart R, Pearce LA: Oral anticoagulants versus antiplatelet therapy for preventing stroke in patients with non-valvular atrial fibrillation and no history of stroke or transient ischemic attacks. Cochrane Database Syst Rev 2007;3:CD006186.

6 Wolf PA, Abbott RD, Kannel WB: Atrial fibrillation: a major contributor to stroke in the elderly. The Framingham Study. Arch Intern Med 1987;147:1561-1564.

7 Rosamond W, Flegal K, Furie K, Go A, Greenlund K, Haase N, et al: Heart disease and stroke statistics - 2008 update: a report from the American Heart Association Statistics Committee and Stroke Statistics Subcommittee. Circulation 2008;117:e25-e146.

8 Jabaudon D, Sztajzel J, Sievert K, Landis T, Sztajzel R: Usefulness of ambulatory 7-day ECG monitoring for the detection of atrial fibrillation and flutter after acute stroke and transient ischemic attack. Stroke 2004;35: 1647-1651.

-9 Fennis TF, Compter A, van den Broek MW, Koudstaal PJ, Algra A, Koehler PJ: Is isolated aphasia a typical presentation of presumed cardioembolic transient ischemic attack or stroke? Cerebrovasc Dis 2013;35:337340.

10 Croquelois A, Bogousslavsky J: Stroke aphasia: 1,500 consecutive cases. Cerebrovasc Dis 2011;31:392-399.

11 Engelter ST, Gostynski M, Papa S, et al: Epidemiology of aphasia attributable to first ischemic stroke: incidence, severity, fluency, etiology, and thrombolysis. Stroke 2006;37:1379-1384.

12 Lamassa M, Di Carlo A, Pracucci G, et al: Characteristics, outcome, and care of stroke associated with atrial fibrillation in Europe: data from a multicenter multinational hospital-based registry (The European Community Stroke Project). Stroke 2001;32:392-398.

13 Tsouli S, Kyritsis AP, Tsagalis G, Virvidaki E, Vemmos KN: Significance of aphasia after first-ever acute stroke: impact on early and late outcomes. Neuroepidemiology 2009;33:96-102.

14 Inatomi Y, Yonehara T, Omiya S, Hashimoto Y, Hirano T, Uchino M: Aphasia during the acute phase in ischemic stroke. Cerebrovasc Dis 2008;25:316-323.

15 Levine RL, Dulli DA, Dixit S, Hafeez F, Khasru M: Isolated Broca's area aphasia and ischemic stroke mechanism. J Stroke Cerebrovasc Dis 2003;12:127-131. 
16 Bang OY, Heo KG, Kwak Y, Lee PH, Joo IS, Huh K: Global aphasia without hemiparesis: lesion analysis and its mechanism in 11 Korean patients. J Neurol Sci 2004;217:101-106.

17 Higgins P, MacFarlane PW, Dawson J, McInnes GT, Langhorne P, Lees KR: Noninvasive cardiac event monitoring to detect atrial fibrillation after ischemic stroke: a randomized, controlled trial. Stroke 2013;44:25252531.

18 Arboix A, Garcia-Eroles L, Massons JB, Oliveres M, Pujades R, Targa C: Atrial fibrillation and stroke: clinical presentation of cardioembolic versus atherotrombotic infarction. Int J Cardiol 1999;73:33-42.

19 Capmany RP, Arboix A, Casanas-Munoz R, Anguera-Ferrando N: Specific cardiac disorders in 402 consecutive patients with ischaemic cardioemboli stroke. Int J Cardiol 2003;95:129-134. 